\title{
INTEGRACIÓN FINANCIERA Y COSTO DE CAPITAL PROPIO EN LATINOAMÉRICA Samuel Mongrut*
}

Instituto Tecnológico y de Estudios Superiores de Monterrey, Campus Querétaro, Centro de Investigación de la Universidad del Pacífico (CIUP)

Darcy Fuenzalida

Departamento de Industrias, Universidad Técnica Federico Santa María, Chile Juan Diego Carrillo

Departamento de Inversiones, Enfoca, Lima-Perú

Luis Alberto Gamero

Departamento de Inversiones, Enfoca, Lima-Perú

(Recibido 26 de abril 2011, aceptado 31 de julio 2011)

\begin{abstract}
Resumen
En este estudio se evalúa la importancia que tiene el nivel de integración financiera sobre los costos de capital propio que enfrentan las empresas en los mercados Latinoamericanos. Se estudia esta relación mediante un modelo no balanceado de datos de panel aplicado a 270 empresas que cotizaron en seis mercados Latinoamericanos (Argentina, Brasil, Chile, Colombia, México y Perú) durante los años 2000 y 2009. Se encontró que una mayor integración financiera ayuda en la disminución de los costos de capital propio para las empresas de la región con un rezago de seis meses a un año. Asimismo se encontró que las empresas Latinoamericanas de mayor tamaño son aquellas que enfrentan un costo de capital propio más bajo; que un aumento en el riesgo país aumenta el costo de capital propio y que un incremento en las expectativas inflacionarias incrementa el costo de capital propio. Los resultados encontrados son robustos ante medidas alternativas de integración financiera.
\end{abstract}

\section{Abstract}

In this paper is assessed the importance of the level of financial integration in the costs of capital that the firms in the Latin American markets face. This relation is studied by means of a non balanced panel data model applied to 270 firms trading in six Latin American markets (Argentina, Brazil, Chile, Colombia, Mexico and Peru) during 2000 and 2009. It was found that a greater financial integration leads to a diminution of the costs of capital for the firms in the region with a lag from six months to one year. Also it was found that the larger Latin American firms face a lower cost of capital; that an increase in the risk country raises the cost of capital and that an increase in the inflationary expectations raises the cost of capital. The obtained results are robust by using alternative measures of financial integration.

Clasificación JEL: G12, F36

Palabras clave: Integración financiera, Costo de capital propio

* Escuela de Graduados en Administración, ITESM, Av. Epigmenio González 500, Fracc. San Pablo, C.P. 76130, Querétaro, Qro, México. Correo electrónico: smongrut@itesm.mx

Samuel Mongrut y Darcy Fuenzalida agradecen al Fondo Nacional de Ciencia y Tecnología (FONDECYT) y al Comité de Investigación y Tecnología (CONICYT) de Chile por financiar este proyecto de investigación (No. 1090440). Asimismo, los autores desean agradecer a los dos árbitros anónimos que con sus comentarios permitieron mejorar el documento. 


\section{Introducción}

Desde inicios de los años noventa los países Latinoamericanos propiciaron cambios tanto regulatorios como políticos que permitieron la apertura de las economías de la región al mundo. En el año 1990 el influjo neto de inversión directa extranjera fue de 8 billones de dólares; mientras que en el año 2008 fue de 129 billones de dólares (Banco Mundial, 2009). Esta situación trajo consigo mayores opciones de financiamiento para las empresas sobre todo en los mercados de capitales donde la capitalización bursátil paso de 77 billones de dólares en 1990 a 2751 billones de dólares en el año 2010 (Banco Mundial, 2009).

Por otra parte, las economías de la región Latinoamericana poseen algunas características que la hacen especialmente atractiva para inversionistas internacionales tales como sus tasas de crecimiento que, en promedio, ha experimentado un crecimiento promedio anual de $6 \%$ durante los últimos 6 años. Otra característica interesante de la región ha sido la reducción del riesgo político, económico y financiero gracias a las políticas de inserción internacional implementadas por los gobiernos a finales de los noventa (Fuenzalida, Mongrut y Nash, 2005). Un claro ejemplo de esta situación es la calificación de grado de inversión con el que cuenta la deuda soberana de Brasil, Chile, México y Perú.

Se denomina integración financiera al proceso mediante el cual se dan cambios regulatorios y políticos para que los mercados financieros de los diferentes países se encuentren más entrelazados. Específicamente, se eliminan barreras directas e indirectas para que instituciones financieras e inversionistas externos operen en el ámbito local y viceversa en los mercados financieros. El mero acto de eliminar barreras se suele denominar liberalización financiera; mientras que integración financiera constituye un proceso gradual (Henry, 2000). Es importante tener presente, que un proceso de liberalización y de integración financiera suele ser motivado por medidas que favorecen la Inversión Directa Extranjera (liberalización económica) y un conjunto de acuerdos entre países que permite el libre flujo de bienes y servicios (integración económica).

En el contexto del mercado de capitales, el término integración financiera implica que dos activos financieros que cotizan en distintos mercados, si poseen el mismo riesgo, deberían comandar el mismo rendimiento esperado. Pretorius (2002) menciona que existen tres categorías que explican el co-movimiento entre los mercados de capitales: el contagio financiero; la integración económica y las características propias de los mercados de capitales, como son la similitud de industrias, la volatilidad y el tamaño de dichos mercados.

Con respecto a la integración económica, no solo se han firmado muchos tratados de libre comercio bilaterales entre países de la región Latinoamericana con China, Estados Unidos de Norteamérica y la Unión Europea sino que el volumen negociado por los países Latinoamericanos con las distintas partes del mundo, expresado como porcentaje del Producto Interno Bruto (PIB), se ha incrementado de un 32\% en 1990 a 48\% en el año 2008 (Banco Mundial, 2009). Más aun, en términos de integración financiera, el stock total de activos y pasivos extranjeros como porcentaje del PIB se ha incrementado, en promedio, de 
un $16 \%$ en el año 2000 a un $18 \%$ en el año 2009 para los seis países considerados en este estudio.

Todo lo anterior indica un mercado regional en expansión, con menores riesgos, con mercados financieros en crecimiento y dinámicos y con mercados de bienes y servicios que se encuentran cada más integrados al mundo. Ante este panorama, la pregunta crucial es si la mayor integración financiera de la región Latinoamericana con el mundo se está reflejando en menores costos de capital requeridos por los inversionistas a las empresas que cotizan en los mercados de capitales Latinoamericanos. La pregunta anterior es particularmente importante ya que de ser así, existiría un incentivo para que las empresas que cotizan en los mercados bursátiles Latinoamericanos incrementen sus inversiones en la región debido a que el costo del financiamiento se estaría reduciendo.

Bekaert y Harvey $(2002,2003)$ indican que en la medida que ingresa un mayor flujo de capitales externo en el mercado de capitales local el precio de las acciones subirá reduciéndose así el rendimiento esperado y como consecuencia también el costo de capital propio. Naturalmente, una mayor integración financiera favorece este mayor ingreso de capitales.

Agenor (2003) analiza la tendencia de integración financiera para países desarrollados y emergentes. Menciona que las economías emergentes han seguido un proceso acelerado de integración desde la década de los 80 y que esto se debe principalmente a la búsqueda de mayor diversificación y mayores tasas de retorno por parte de los inversionistas, ayudados por la liberalización de las economías y la disminución de restricciones sobre los flujos de dinero.

Una primera evidencia de que el costo de capital propio se está reduciendo en Latinoamérica a inicios del siglo XXI es proporcionada por Fuenzalida y Mongrut (2010). Estos autores estiman costos de capital propio para seis mercados Latinoamericanos durante el periodo 2000-2005 utilizando hasta diez modelos distintos propuestos por la literatura actual. En ellos evidencian que los costos de capital propio se están reduciendo, pero no muestran la relación entre este comportamiento y el proceso de integración financiera.

El principal objetivo de este estudio consiste en mostrar que la mayor integración financiera de los mercados Latinoamericanos efectivamente está reduciendo el costo de capital propio en los países de la región identificando otros factores que también pueden afectar el costo de capital propio.

El documento está organizado en cuatro secciones, además de la introducción. En la segunda sección se analizan los indicadores de integración financiera utilizados en la literatura, los determinantes del costo de capital propio encontrados en la literatura y la evidencia empírica encontrada en otras regiones sobre la relación entre el costo de capital propio y el proceso de liberalización e integración financiera.

En la tercera sección se explica la metodología utilizada para estimar los costos de capital propio y los indicadores de integración financiera y se describe 
el modelo econométrico utilizado para interrelacionar ambas variables. En la cuarta sección, se comentan los resultados obtenidos y en la última sección se concluye el trabajo.

\section{Revisión de la literatura}

\subsection{Indicadores de integración financiera}

Uno de los aspectos más importantes del presente estudio es seleccionar los indicadores de integración financiera que serán relacionados con el costo de capital propio. La Tabla 1 muestra una síntesis de principales indicadores de integración financiera sobre la base de cuatro estudios principales que fueron seleccionados debido a que analizan diferentes indicadores de integración financiera.

Tabla 1. Indicadores de integración financiera

\begin{tabular}{|c|c|}
\hline Autores & Indicadores de Integración Financiera \\
\hline $\begin{array}{l}\text { Adam et al. } \\
(2002)\end{array}$ & $\begin{array}{l}>\text { Diferenciales entre las tasas de interés de los países } \\
>\text { Porcentaje de bancos extranjeros } \\
>\text { Evolución de activos extranjeros de los bancos locales } \\
>\text { Porcentaje de acciones administradas por fondos de } \\
\text { inversión con una estrategia de inversión internacional }\end{array}$ \\
\hline $\begin{array}{l}\text { Prasad et al. } \\
(2003)\end{array}$ & $\begin{array}{l}>\text { Liberalización en el control de capitales } \\
\text { Stock de activos y pasivos extranjeros como porcentaje del } \\
\text { PIB }\end{array}$ \\
\hline $\begin{array}{l}\text { Lane y Milesi- } \\
\text { Ferretti (2003) }\end{array}$ & $\begin{array}{l}\text { Stock de activos y pasivos extranje ros como porcentaje del } \\
\text { PIB } \\
\text { Stock de activos y pasivos en acciones e inversión } \\
\text { extranjera directa como \% del PBI }\end{array}$ \\
\hline Vo (2005) & $\begin{array}{l}\text { Stock de activos y pasivos extranje ros como porcentaje del } \\
\text { PIB } \\
>\text { Total de flujos de capital extranjero como porcentaje del PIB } \\
\text { Stock total de inversión extranjera dire cta (IED) y el total de } \\
\text { inversiones extranjeras en acciones como porcentaje del } \\
\text { PBI }\end{array}$ \\
\hline
\end{tabular}

Fuente: elaboración propia

Adam, Jappelli, Menichini, Padula y Pagano (2002) analizan indicadores para medir el grado de integración financiera de los mercados de capitales de la Unión Europea (UE) y proponen un conjunto de indicadores para monitorear la integración financiera de la UE en el futuro. Estos autores encuentran que los indicadores de los mercados de créditos, bonos y de acciones indican un mayor grado de integración financiera para los países de la UE; mientras que los indicadores basados en las decisiones de los hogares y las firmas (p.e. la correlación en la variación porcentual del consumo entre países), revelan que todavía el nivel de distribución de riesgos es bajo para los países de la UE. Asimismo los indicadores de diferencias institucionales muestran un obstáculo para la integración financiera de la UE.

Prasad, Rogoff, Wie y Kose (2003) buscan encontrar la relación entre integración financiera y crecimiento económico, específicamente desean averiguar 
el impacto de la integración financiera sobre la volatilidad macroeconómica y los factores que pueden ayudar a aprovechar los beneficios de la integración financiera. Estos autores no encontraron que el mayor grado de integración financiera genere crecimiento económico, pero encontraron que un sólido marco macroeconómico y el desarrollo de los mercados financieros locales son estrategias adecuadas para buscar una mayor integración. Los autores favorecen la utilización del stock de pasivos y activos extranjeros como porcentaje del PIB como indicador de integración financiera.

Lane y Milesi-Ferretti (2003) analizan las principales características de la integración financiera en las últimas dos décadas y relaciona el crecimiento de activos y pasivos externos como causantes de la integración. Estos autores encuentran que variables como apertura comercial, PBI per cápita y capitalización bursátil son exitosas para explicar la variación del grado de integración financiera de un país y que los retornos sobre activos y pasivos externos dependen de la composición entre activos de capital ya que estos son los que han generado mayores retornos en los últimos años.

Vo (2005) investiga los principales determinantes de la integración financiera considerando una serie de características de cada país tales como controles de capital, grado de desarrollo económico, situación política, profundidad de los mercados financieros, entre otros. Encuentra que algunos de los indicadores utilizados para determinar el grado de integración financiera de un país no son significativos y concluye que las variables de control de capitales, apertura al comercio internacional, mercado de créditos local y crecimiento económico son factores que ayudan a explicar la variación del grado de integración financiera.

Como se puede observar, un indicador común en los estudios reseñados es el stock de activos y pasivos extranjeros como porcentaje del PIB que es un indicador que toma en cuenta todos los tipos de inversión extranjera hechas por inversionistas internacionales en el país. Otra característica común entre los estudios reseñados es que la mayoría considera una combinación de indicadores stock e indicadores flujo. En este estudio se consideraran como medidas de integración financiera el stock de activos y pasivos extranjeros como porcentaje del PIB (indicador stock) y el flujo de capital extranjero como porcentaje del PIB (indicador flujo).

\subsection{Factores que afectan el costo de capital propio}

En la literatura financiera se han identificado los factores que afectan los rendimientos accionarios. En un trabajo seminal, Fama y French (1992) mostraron que el ratio de valor en libros sobre valor de mercado (BM) y el tamaño en función de la capitalización bursátil son dos factores que afectan los rendimientos de portafolios accionarios. Específicamente, los portafolios compuestos por empresas de mayor tamaño ofrecen rendimientos inferiores a los portafolios compuestos por empresas de menor tamaño. Más aun, los portafolios compuestos por empresas con un bajo ratio BM ofrecen también rendimientos inferiores que los portafolios compuestos por empresas con un alto ratio BM.

Omran y Pointon (2004) encontraron algunos de los principales determinantes de los costos de capital propios para mercados emergentes, centrándose 
en el caso de Egipto. Encontraron que los costos de capital para las compañías listadas son fuertemente influenciados por el tamaño de la empresa, el ratio $\mathrm{BM}$, la liquidez de las acciones y por los riesgos de mercado.

Por otro lado, Hail y Leuz (2006) analizan la influencia de la efectividad de las instituciones legales y las regulaciones sobre los costos de capital propio para 40 países durante los años 1992-2001. Estos autores buscaron encontrar si es que las diferencias entre las regulaciones de los mercados de capitales se encuentran relacionadas con las diferencias en los costos de capital propio entre los diferentes países de la muestra. Encontraron que aquellos países donde el mercado de capitales está más regulado y las instituciones legales son más fuertes se tienen costos de capital propio inferiores a aquellos reportados en los países donde el mercado de capitales esta menos regulado y las instituciones legales son más débiles.

Chen, Chen y Wei (2009) analizaron los efectos del gobierno corporativo, la protección a los inversionistas y la calidad de las instituciones legales sobre los costos de capital propio. Los autores concluyeron que las empresas con el peor gobierno corporativo ofrecen los costos de capital propio más altos y que este efecto se acentúa si las instituciones legales son débiles. Asimismo, encontraron que la protección a los inversionistas puede actuar como un reemplazo de un buen gobierno corporativo.

A partir de los estudios reseñados se puede concluir que factores tales como el tamaño de las empresas, el ratio BM, la liquidez de las acciones, las regulaciones de los países en materia de protección de los inversionistas y del mercado de capitales, la fortaleza de las instituciones legales y el buen gobierno corporativo de las empresas son factores que influyen en la magnitud de los costos de capital propio.

\subsection{Relación entre la integración financiera y el costo de capital propio}

La relación entre la integración financiera y el costo del capital propio ha sido abordada desde diversos puntos de vista. Uno de los primeros estudios sobre el tema es el de Bekaert y Harvey (1995) donde los autores se pregunta si la integración financiera de los mercados de capitales varía en el tiempo. Para responder esta pregunta los autores se apoyan de un modelo de cambio de régimen donde si un mercado de capitales está completamente segmentado el costo de capital propio viene dado por el CAPM en su versión local y en el caso en el que este muy integrado el costo de capital propio viene dado por una versión global del CAPM. A partir del este modelo, los autores proponen una medida de integración financiera y concluyen que algunos mercados de capitales están más integrados de lo que las medidas de liberalización financiera indican y otros menos integrados.

Henry (2000) analizó el efecto de la liberalización financiera sobre los índices bursátiles accionarios de doce economías emergentes. Encontró que los índices bursátiles accionarios ofrecían un rendimiento anormal del 3\% mensual alrededor de una ventana de ocho meses del anuncio de la primera medida de 
liberalización financiera, lo cual es consistente con una reducción del costo de capital propio.

Bekaert y Harvey (2000) buscan una relación empírica entre distintas medidas de liberalización financiera de los mercados de capitales y los costos de capital. La liberalización de los mercados de capitales se midió a través de tres indicadores principales: la introducción de American Depositary Receipts (ADRs), el levantamiento de restricciones a la inversión privada y el flujo de inversión estadounidense en los mercados emergentes. Los autores hallaron que el ingreso de capitales extranjeros en el mercado de capitales local produce un incremento en el precio de las acciones y una reducción en el costo de capital entre 5 y 75 puntos básicos. No obstante, la condición para que esto ocurra es que la correlación entre los retornos de los mercados de capitales local y mundial sea baja.

Kim y Singal (2000) estudiaron el efecto que tendría el permitir que inversionistas internacionales ingresen en el mercado local sobre los rendimientos accionarios. Los autores encontraron que la liberalización financiera de los mercados de capitales aumentaba el precio de las acciones domésticas sin que aumente la volatilidad de las mismas; esto producía a una disminución del retorno de las acciones y por ende una disminución en el costo de capital propio que enfrentan las empresas locales.

Errunza y Miller (2000) estudiaron el efecto de la liberalización financiera sobre el costo de capital utilizando una muestra de 126 empresas en 32 países. Encontraron que la habilidad de selección de títulos de los inversionistas norteamericanos, antes de que estos títulos coticen en el mercado estadounidense, es el principal factor por el cual el costo de capital se redujo hasta en un $42 \%$.

Bekaert, Harvey y Lumsdaine (2001) buscan precisar de forma empírica la fecha de la integración financiera entre los mercados locales con el mercado mundial y para ello en lugar de identificar las fechas de los cambios regulatorios buscan un quiebre estructural en las series financieras de los rendimientos accionarios y por dividendos. Al hacerlo encuentran que las fechas encontradas no corresponden siempre con las fechas de los cambios regulatorios, pero que los rendimientos por dividendos son menores posteriormente al quiebre estructural.

Jain (2005) analizó el impacto del anuncio e introducción efectiva de los mercados electrónicos, como medida de liberalización financiera, sobre el costo de capital propio en 120 países y encontró una disminución significativa en loa rendimientos esperados, especialmente en el caso de los países en vías de desarrollo. Su conclusión es que el mercado electrónico promueve la liquidez al reducirse los costos de transacción y por consiguiente el costo de capital propio disminuye.

A lo largo de los estudios reseñados se puede observar un gran interés por entender cuáles son los efectos de la apertura de los mercados de capitales sobre los costos de capital propio. Diversas regiones han sido estudiadas para 
entender este fenómeno y se puede comprobar que la gran mayoría de los estudios concuerdan en que la integración financiera de un país conlleva a una disminución de los costos de capital propio que enfrentan las empresas.

\section{Metodología}

La metodología utilizada considera dos etapas claramente identificadas, primero se estiman los costos de capital propio para cada país de acuerdo con un modelo planteado por la literatura; segundo se estiman los indicadores de integración financiera y finalmente de aplica un modelo no balanceado de datos de panel para poder verificar la hipótesis sobre si una mayor integración genera menores costos de capital propio. A continuación se explica con mayor detalle cada etapa.

\subsection{Estimación de costos de capital propio}

Estudios anteriores han estimado el costo de capital propio con diversos modelos alternativos. La Tabla 2 muestra un resumen de los principales estudios sobre el tema aplicados a distintos grupos de países emergentes.

Tabla 2. Estimaciones del costo de capital propio en diversas regiones emergentes

\begin{tabular}{|c|c|c|}
\hline Autores & Región & Modelos utilizados \\
\hline Jain (2005) & Mundial & $\begin{array}{c}\text { Variante del Capital Asset Pricing } \\
\text { Model. ICAPM }\end{array}$ \\
\hline Hail y Leuz (2006) & Mundial & Variantes del FCD o del MDD \\
\hline $\begin{array}{lll}\text { Collins } & \text { y } & \text { Abrahamson } \\
(2005) & & \end{array}$ & Africana & Variantes delCAPM: D-CAPM \\
\hline Ameer (2007) & Asiática & $\begin{array}{l}\text { Modelos de Pom erleano y Zhan g } \\
\text { (1999) y de Goedhart, Koller y } \\
\text { Williams (2002) }\end{array}$ \\
\hline $\begin{array}{l}\text { Mongrut, Paškevičius, } \\
\text { Dubinskas, Kovalevskaja y } \\
\text { Fuenzalida (2010) }\end{array}$ & Báltica & Variantes del CAPM \\
\hline $\begin{array}{l}\text { Fuenzalida y } \text { Mongrut } \\
(2010)\end{array}$ & Latinoamericana & Variantes del CAPM \\
\hline
\end{tabular}

Fuente: elaboración propia

En el estudio ya referido de Jain (2005) se utiliza la variante internacional del Modelo de Equilibrio de activos Financieros (CAPM, por sus siglas en inglés) aplicado a 120 países del mundo. Por su parte el estudio de Hail y Leuz (2006) utiliza como estimación del costo de capital propio el promedio de cuatro modelos basados en el flujo de caja descontado (FCD) o en el modelo de dividendos descontados (MDD).

Collins y Abrahamson (2005) estimaron los costos de capital propio en diez sectores y en seis países africanos (Egipto, Kenia, Marruecos, Namibia, África del Sur y Zimbabue) utilizando variantes del CAPM tales como el modelo de equilibrio de activos financieros con riesgo de pérdida o downside (D-CAPM, por sus siglas en inglés) planteado por Estrada (2002). Estos autores encontraron, 
para el periodo 1995-2002, que el costo de capital propio de los mercados de capitales considerados había disminuido debido a una mayor liberalización e integración financiera.

Ameer (2007) estudió la variación de los costos de capital propio de 242 empresas en 6 países emergentes asiáticos (India, Indonesia, Malasia, Pakistán, Tailandia y Corea del Sur) para el periodo 1990-2004. Sin embargo, en lugar de utilizar los modelos de estimación basados en el CAPM, el autor utilizó medidas alternativas basadas en las propuestas de Pomerleano y Zhang (1999) y Goedhart, Koller y Williams (2002). Encontró que la disminución de los costos de capital propio está asociada a una mayor liberalización financiera de los mercados de capitales asiáticos, aunque con diferencias importantes entre países.

Mongrut, Paškevičius, Dubinskas, Kovalevskaja y Fuenzalida (2010) estudiaron el caso de los tres países bálticos (Estonia, Lituania y Latvia) para el período 2005-2008 y utilizando tres variantes del CAPM: El D-CAPM, el modelo de Mariscal y Lee (1993) y el modelo de Damodaran (2003). Los autores encontraron que los costos de capital propio se han incrementado durante este período influenciados por el riesgo de la región y concluyen que la región báltica se encuentra altamente expuesta a la inestabilidad financiera internacional debido a la falta de un sistema financiero y económico adecuado.

El estudio que más modelos de capital propio utiliza es el de Fuenzalida y Mongrut (2010). Estos autores utilizan siete variantes del CAPM planteadas por la literatura actual para analizar el comportamiento de los costos de capital propio en seis países Latinoamericanos (Argentina, Brasil, Colombia, Chile, México y Perú) durante el periodo 2000-2005. Asimismo, utilizan dos modelos ad hoc para inversionistas imperfectamente diversificados y el modelo de Erb, Harvey y Viskanta (1996) para inversionistas no diversificados. Los autores encontraron que ningún modelo de costo de capital propio de integración parcial era mejor que otro en términos de la magnitud estimada y que los costos de capital propio han disminuido durante los primeros cinco años del siglo XXI para los países de América Latina.

A partir de los estudios reseñados se puede concluir que los autores suelen utilizar, como aproximaciones del costo de capital propio, a variantes del CAPM, a modelos basados el flujo de caja descontado o en el modelo de dividendos descontados (que utilizan proyecciones de analistas) y a modelos ad hoc que no poseen fundamentos teóricos sólidos.

En este estudio se utiliza el modelo de Damodaran (2003) como aproximación del costo de capital propio debido a que es una variante del CAPM que posee fundamentos teóricamente sólidos (Fuenzalida y Mongrut, 2010) y es el modelo más utilizado por profesores, analistas y compañías (Fernández, Aguirreamalloa y Corres, 2011). Damodaran (2003) propuso que el costo de capital propio para un mercado emergente podía ser estimado asumiendo una situación de integración parcial y un equilibrio entre los rendimientos ajustados por riesgo del mercado local de acciones y de bonos. Este último supuesto es 
particularmente útil debido a que permite evitar el cálculo de una prima por riesgo local que suele ser negativa en mercados emergentes y más aún, cuando un error en la estimación de la prima por riesgo representa más del $90 \%$ del error de estimación en el costo de capital propio (Ferson y Locke, 1998).

El modelo de Damodaran (2003) está representado por la siguiente ecuación:

$$
E\left(R_{i}\right)=R_{f}^{U S}+\beta_{i}^{U S}\left(R_{m}^{U S}-R_{f}^{U S}\right)+\lambda_{i}\left(R_{B}^{M E}-R_{B}^{U S}\right)\left(R_{B}^{M E}-R_{B}^{U S}\right)\left(\frac{\sigma_{m}^{M E}}{\sigma_{B}^{M E}}\right)
$$

donde:

$R_{f}^{U S}$ : Rendimiento de los bonos del tesoro americano

$\beta_{i}^{U S}$ : Riesgo sistemático de una empresa comparable a la empresa "i" en el mercado americano

$R_{m}^{U S}-R_{f}^{U S}:$ Prima por riesgo de mercado americana

$\lambda_{i}$ : Exposición al riesgo país de la compañía "i"

$R_{B}^{M E}-R_{B}^{U S}$ : Riesgo de incumplimiento del país (se aproxima con el diferencial extrapolado del Emerging Markets Bond Index (EMBI+) del país)

$\sigma_{m}^{M E} / \sigma_{B}^{M E}:$ Ratio de volatilidad relativa

$\left(R_{B}^{M E}-R_{B}^{U S}\right) \sigma_{m}^{M E} \sigma_{B}^{M E}:$ Prima por riesgo país

Si bien, bajo los supuestos planteados por Damodaran, la expresión anterior es teóricamente sólida, siempre que la integración financiera parcial de los mercados de capitales sea aditiva, la aplicación empírica de la formula enfrenta el problema de la elevada volatilidad de los mercados de capitales emergentes para la estimación del ratio de volatilidad relativa.

Con la finalidad de evitar el problema de excesiva volatilidad es posible asumir que todas las empresas poseen la misma sensibilidad al riesgo país con lo cual lambda sería igual a la unidad. De la misma manera se puede asumir que el ratio de volatilidad relativa en períodos de estabilidad es igual a 1.5; Walker (2003) denomina a este supuesto la conjetura de Damodaran. Al imponer estos supuestos la ecuación anterior queda especificada de la siguiente manera:

$$
E\left(R_{i}\right)=R_{f}^{U S}+\beta_{i}^{U S}+\left(R_{m}^{U S}-R_{f}^{U S}\right)+\left(R_{B}^{M E}-R_{B}^{U S}\right)(1.50)
$$

La ventaja de esta especificación es que permite con magnitudes de costos de capital propio que son razonables (inferiores al 100\%) manteniendo la heterogeneidad de los resultados entre países. La desventaja es que esta especificación no permite trabajar con sectores económicos debido a que la exposición al riesgo país de todas las empresas se asume igual a la de todo el mercado en su conjunto. 
La ecuación 2 se estima trimestralmente para cada empresa considerada en la muestra y perteneciente a cada uno de los seis países considerados entre los años 2000 y 2009. Los betas son estimados de forma mensual y con una ventana móvil de cinco años que se actualiza mensualmente, aunque se considera únicamente el valor encontrado para cada trimestre.

Así para estimar los costos de capital propios correspondientes al primer trimestre del año 2000, estos se estimaron con betas que fueron calculados para el periodo que va desde el segundo trimestre de 1995 al primer trimestre del 2000. La Tabla 3 muestra las estadísticas descriptivas de los costos de capital propio estimados con la ecuación 2 y los valores constituyen promedios simples de los costos de capital para las empresas de cada país.

Table 3. Estadística de los costos de capital propio para Latinoamérica

\begin{tabular}{ccccccc}
\hline \multicolumn{7}{c}{ Damodaran } \\
\hline 2000 & $20.9 \%$ & $22.6 \%$ & $13.6 \%$ & $17.3 \%$ & $21.0 \%$ & $17.7 \%$ \\
2001 & $31.0 \%$ & $22.9 \%$ & $10.3 \%$ & $14.5 \%$ & $17.9 \%$ & $16.4 \%$ \\
2002 & $92.2 \%$ & $28.2 \%$ & $9.1 \%$ & $15.7 \%$ & $14.6 \%$ & $14.7 \%$ \\
2003 & $89.8 \%$ & $19.3 \%$ & $7.9 \%$ & $12.3 \%$ & $11.2 \%$ & $10.7 \%$ \\
2004 & $86.0 \%$ & $16.2 \%$ & $7.9 \%$ & $11.6 \%$ & $10.8 \%$ & $11.0 \%$ \\
2005 & $49.8 \%$ & $17.2 \%$ & $7.6 \%$ & $11.9 \%$ & $12.2 \%$ & $11.1 \%$ \\
2006 & $14.3 \%$ & $15.4 \%$ & $8.2 \%$ & $16.0 \%$ & $13.2 \%$ & $9.5 \%$ \\
2007 & $14.1 \%$ & $13.9 \%$ & $9.0 \%$ & $15.4 \%$ & $13.3 \%$ & $9.4 \%$ \\
2008 & $20.6 \%$ & $13.2 \%$ & $10.9 \%$ & $13.2 \%$ & $12.9 \%$ & $12.6 \%$ \\
2009 & $25.3 \%$ & $11.8 \%$ & $9.7 \%$ & $11.3 \%$ & $14.1 \%$ & $12.6 \%$ \\
\hline Min & $14.1 \%$ & $11.8 \%$ & $7.6 \%$ & $11.3 \%$ & $10.8 \%$ & $9.4 \%$ \\
Max & $92.2 \%$ & $28.2 \%$ & $13.6 \%$ & $17.3 \%$ & $21.0 \%$ & $17.7 \%$ \\
Media & $44.4 \%$ & $18.1 \%$ & $9.4 \%$ & $13.9 \%$ & $14.1 \%$ & $12.6 \%$ \\
Mediana & $28.2 \%$ & $16.7 \%$ & $9.1 \%$ & $13.8 \%$ & $13.3 \%$ & $11.8 \%$ \\
Desv.Est. & 0.326 & 0.052 & 0.018 & 0.021 & 0.032 & 0.028 \\
\hline
\end{tabular}

Fuente: elaboración propia

\subsection{Estimación de los indicadores de integración financiera}

Para seleccionar los indicadores de integración financiera de cada país se tomó como referencia algunas de las recomendaciones de Lane y Milesi-Ferretti(2003), Prasad et. al. (2003) y Vo (2005). Estos autores coinciden en recomendar al ratio de stock de activos y pasivos extranjeros sobre el PIB como aproximación adecuada para medir el nivel de integración financiera de un país. Asimismo se tomó como indicador complementario al ratio de los flujos de capital sobre el PIB ya que este indicador se enfoca en la variación de los niveles de integración financiera en el corto plazo (Vo, 2005). De esta forma, los indicadores de integración financiera (IF) se calculan como sigue: 


$$
I F_{1}=\frac{S A E+S P E}{P I B}
$$

donde:

$I F_{1}$ : Stock del total de activos y pasivos extranjeros como porcentaje del PIB $S A E$ : Stock de activos extranjeros $S P E$ : Stock de pasivos extranjeros

$$
I F_{2}=\frac{F C E}{P I B}
$$

donde:

$I F_{2}$ : Flujo de capital extranjero como porcentaje del PIB

$F C E$ : Flujo de capital extranjero

Con la finalidad de verificar si ambos indicadores eran redundantes se realizó la prueba de correlación de Spearman (no reportada) entre ambos indicadores para cada uno de los seis países considerados. Se rechazó la hipótesis nula de independencia para Argentina, Brasil, Chile y Colombia con lo cual la inclusión de ambas variables simultáneamente en el modelo podrían generar un problema de multicolinealidad. Por ello se decidió realizar la estimación sin incluir las dos variables en el mismo modelo; es decir, estimar por separado las influencias de cada uno de estos indicadores sobre los costos de capital propio.

Los gráficos 1 y 2, muestran que ambos indicadores, con excepción de Argentina y en menor grado de México, han seguido una tendencia creciente hasta el 2008, año en el que estalló la crisis financiera mundial y en el cual una gran cantidad de inversionistas extranjeros se retiraron de la región Latinoamericana.

Gráfica 1. Tendencia $I F I_{1}$ Latinoamérica 2000-2009

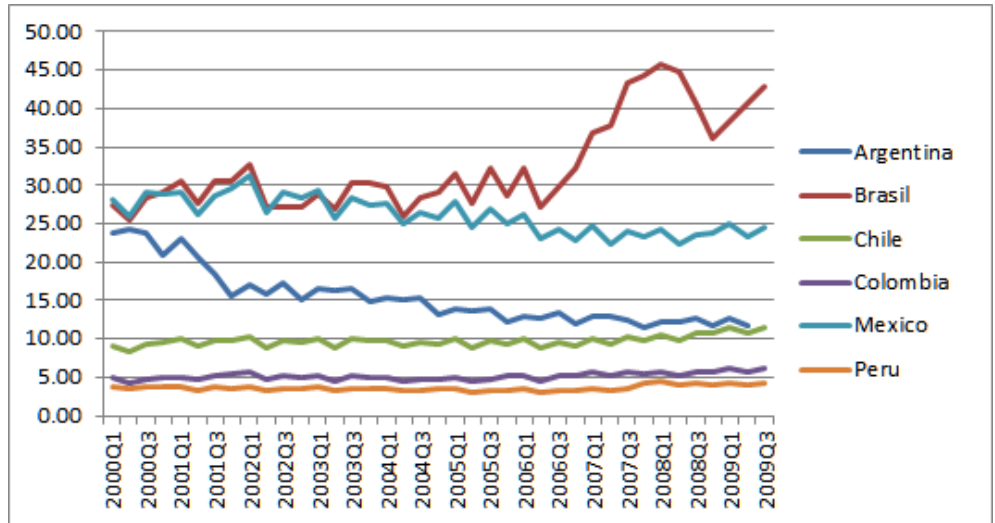

Fuente: IFS, Fondo Monetario Internacional. Elaboración propia. 
Gráfica 2. Tendencia $I F I_{2}$ Latinoamérica 2000-2009

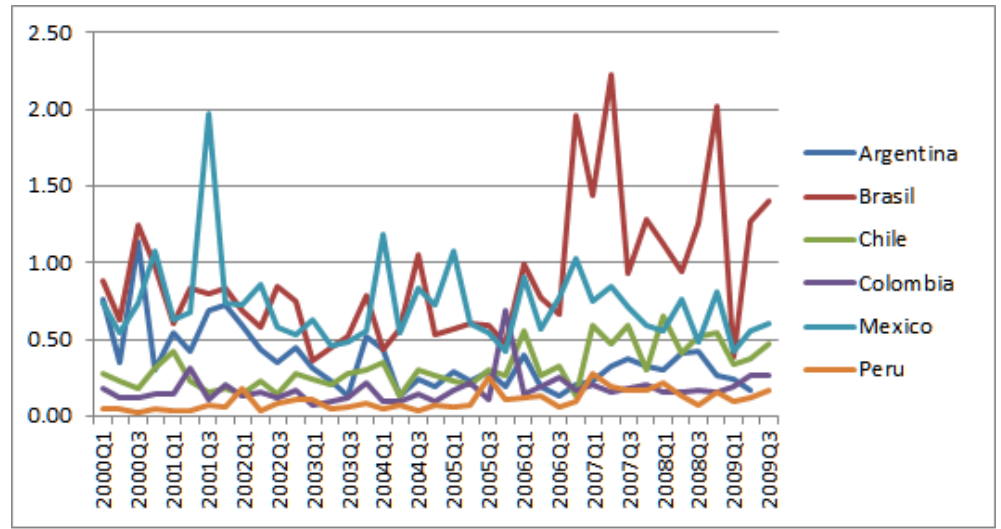

Fuente: IFS, Fondo Monetario Internacional. Elaboración propia.

\subsection{Modelo econométrico}

Con la finalidad de verificar la relación entre los costos de capital propio (CE) y el nivel de integración financiera (IFI) de un país se utilizó un modelo no balanceado de datos de Panel. En este modelo se analizaron los problemas de heterocedasticidad y de autocorrelación. Para analizar el problema de heterocedasticidad se aplicó la prueba modificada de Wald (Wooldridge, 2002). La hipótesis nula de esta prueba acepta la homocedasticidad, es decir, varianza constante para todas las unidades transversales. Esta prueba fue aplicada a todos los países, rechazándose la hipótesis nula y obteniendo como resultado problemas de heterocedasticidad para todos los países.

El problema de autocorrelación también fue analizado con la prueba de Wooldridge (2002). Si es que se acepta la hipótesis nula de esta prueba se acepta la no existencia de autocorrelación; por lo tanto si es que esta se rechaza se aceptará que la estimación presenta este problema. Al aplicar la prueba de Wooldridge para los países latinoamericanos estudiados se encontró que todos presentaban el problema de autocorrelación. Debido a que en todos los países de la muestra se encontraron los problemas de heteroscedasticidad y autocorrelación, y a que se está trabajando con paneles no balanceados, se aplicó el Método de Errores Estándar Corregidos para Panel (Prais-Winsten regression) ya que esta metodología toma en cuenta estos problemas al momento de realizar la estimación y corrige la posibilidad de correlación contemporánea de los errores. Esto permitió obtener mejores estimaciones en cada uno de los modelos. 
En cuanto a las variables de control a utilizar en el modelo, se utilizó como primera variable al riesgo país medido por el índice compuesto (ICRG) del Political Risk Services (PRS) y que está compuesto por tres componentes: riesgo político, riesgo económico y riesgo financiero. Nótese que esta variable de control se incluyó también con la finalidad de considerar los factores institucionales tales como corrupción, burocracia y reglas de juego que resultaron ser importantes en el estudio de Hail y Leuz (2006).

Otra variable de control que se incluyó fue el valor en libros sobre valor de mercado (BM) ya que, según autores como Omran y Pointon (2004), Hail y Leuz (2006) y Chen, Chen y Wei (2009), este indicador representa un determinante importante sobre los costos de capital propio en la medida que muestra las expectativas del mercado financiero acerca del rendimiento de la empresa. Esta variable se calcula como el logaritmo del valor en libro de las acciones (VL) de una empresa entre el valor de mercado (VM) de estas mismas:

$$
L N(B M)=L N\left(\frac{V L}{V M}\right)
$$

El tamaño de la empresa (MC) representa también un determinante importante sobre los costos de capital propio debido a que las empresas más grandes deberían de tener un menor costo de capital propio dadas sus economías de escala en la obtención de financiamiento. Esta variable es medida utilizando como aproximación el logaritmo de la capitalización bursátil de mercado en millones de dólares de cada empresa:

$$
L N(M C)=\text { Capitalización de Mercado (US\$ millones })
$$

Según Omran y Pointon (2004) la liquidez (LIQ) afecta al costo de capital propio ya que los retornos esperados de las acciones deben ser mayores para acciones menos líquidas que para acciones más liquidas, esto para compensar los altos costos de transacción. Esta se medirá como el porcentaje negociado de acciones sobre el total de las acciones listadas en bolsa de cada empresa:

$$
L I Q=\frac{\text { Número de acciones negociadas }}{\text { Total de acciones }}
$$

Por otro lado, se consideró también a la inflación (INF) como variable de control ya que esta afecta al costo de capital propio ya que todas las variables que se utilizan para estimarlo están expresadas en términos nominales, asimismo los inversionistas exigirán mayores retornos durante periodos de alta inflación ya que esta reduce la rentabilidad real de los títulos de cada país al estar listados en la moneda local. Esta variable es medida como la variación trimestral del índice de precios al consumidor de cada país.

Por último, para analizar la conveniencia de la utilización de un modelo de efectos fijos o uno de efectos aleatorios se utilizó la prueba de Hausman (1978), la cual analiza la posible correlación entre el componente de error individual y las variables a utilizar a través de las diferencias entre los coeficientes de 
efectos fijos y efectos aleatorios. La hipótesis nula de esta prueba indica que los estimadores de efectos aleatorios y de efectos fijos no difieren sustancialmente y por lo tanto la estimación de efectos aleatorios sería la más eficiente. Por consiguiente, al rechazar la hipótesis nula el modelo más conveniente para el análisis planteado sería el de efectos fijos. Al realizar la prueba de Hausman (no reportada) para la estimación de cada país y se rechazó la hipótesis nula en todos los casos. Este resultado es el esperado ya que existen características específicas de cada empresa que afectan al modelo en el momento de la estimación que serán corregidos al estimarse un modelo de efectos fijos.

Dado lo anterior, el modelo no balanceado de datos de panel a ser estimado para cada país y luego para la región Latinoamericana queda especificado como:

$$
Y_{i j, t}=\infty_{i j, t}+X_{i j, t} \beta_{i j, t}+n_{i j, t}
$$

donde:

$Y_{i j, t}=C E_{i j, t}$

$X_{i j, t}=\left[I F I M_{j, t-k}, \ln I C R G_{i j, t-1}, \ln B M_{i j, t-1}, \ln M C_{i j, t}, L I Q_{i j, t,}, I N F_{i j, t}\right]$

$n_{i j, t}=\gamma_{i j, t}+\varepsilon_{i j, t}$

$M$ : muestra el indicador de integración a utilizar: $I F I_{1}$ o $I F I_{2}$

$K$ : rezago del indicador de integración

$i j, t$ : empresa "i" del país " $j$ " en el trimestre " $t$ "

$\gamma_{i, j}:$ Efecto individual

$\varepsilon_{i, j, t}:$ Perturbación

Es importante remarcar que una ventaja de trabajar con un modelo de panel de datos es que posible controlar por aquellos factores no observables que afectan la respuesta de cada empresa pero que, sin embargo, se mantienen relativamente constantes en el tiempo. Esto último es importante debido a que es difícil capturar todos los factores que afectan a los costos de capital propio.

Adicionalmente el que la variable dependiente sea un costo de capital propio que se estima con error no es necesariamente un problema siempre que la incertidumbre con respecto a la variable dependiente sea constante a través de las observaciones. Si este no es el caso, los errores de la regresión sufrirán de heterocedasticidad, pero como Lewis (2000) indica corregir los errores estándares por este problema con el método de White o uno de dos etapas soluciona razonablemente el problema

Se espera que los indicadores de integración financiera mantengan coeficientes con signos negativos ya que una mayor integración financiera debe resultar en menores costos de capital. Asimismo, se espera que los coeficientes 
estimados para el índice compuesto de riesgo país (ICRG), el tamaño de las empresas (MC) y la liquidez (LIQ) tengan un signo negativo ya que un mayor riesgo país implica un mayor costo de capital propio (aquí nótese que el ICRG es un numero de 0 a 100 y su mayor valor indica menor riesgo país), las empresas de mayor tamaño requerirán un menor costo de capital propio por ser menos riesgosas que las de menor tamaño y las empresas con acciones más liquidas deberán ofrecer un menor costo de capital propio ya que los títulos presentan un menor riesgo al poder ser vendidos en cualquier momento.

Por otra parte, se espera signos positivos para el ratio de valor en libros sobre valor de mercado (BM) y para la inflación esperada. Las acciones con un elevado ratio de $\mathrm{BM}$ son las que no son populares (acciones de valor) y deben ofrecer rendimientos superiores que las acciones con un bajo BM (Fama y French, 1998). Finalmente, una elevada inflación esperada deberá incrementar el costo de capital propio.

Adicionalmente se tomó en cuenta lo que Mongrut et. al. (2010) llaman el sesgo de visión prospectiva (forward-looking bias) que implica que los inversionistas toman la decisión de invertir en un país solo después de conocer el riesgo país del mismo y no al mismo tiempo o antes. Es por esta razón que variables como el índice compuesto de riesgo país (ICRG) y el ratio valor en libros sobre valor de mercado (BM) fueron rezagadas un trimestre.

\section{Análisis de resultados}

Para verificar la hipótesis sobre si una mayor integración financiera disminuye el costo de capital propio en la región Latinoamericana se decidió aplicar un modelo no balanceado de datos de panel para cada uno de los seis países Latinoamericanos (Argentina, Brasil, Colombia, Chile, México y Perú) desde el primer trimestre del año 2000 hasta el cuarto trimestre del año 2009. Se utilizaron las bases de datos de Economatica, Bloomberg, Banco Mundial, Political Risk Services y International Financial Statistics (IFS) del Fondo Monetario Internacional para extraer la información sobre las variables del modelo y se estimaron los modelos con data trimestral debido a la limitación de información mensual para algunas variables.

La base de datos comprende 61 empresas para el caso argentino, 57 empresas para el caso brasilero, 57 empresas para el caso chileno, 23 empresas para el caso colombiano, 41 empresas para el caso mexicano y 31 empresas para el caso peruano, lo cual resulta en un total de 270 empresas analizadas. Por otro lado, es importante mencionar que los rendimientos accionarios fueron calculados en dólares, ajustados por dividendos y de forma compuesta continua.

En la Tabla 4, se muestran los resultados de la estimación de la ecuación 7 utilizando como indicador de integración financiera el stock del total de activos y pasivos extranjeros como porcentaje del PIB $\left(I F I_{1}\right)$. 
Tabla 4. Integración financiera y costos de capital propio en América Latina por países (2000-2009): $I F I_{1}$

\begin{tabular}{|c|c|c|c|c|c|c|c|}
\hline \multirow[b]{2}{*}{ Indicador } & \multicolumn{7}{|c|}{ Modelo de Efectos Fijos con errores Estándares Corregidos (Prais - Winsten) } \\
\hline & Argentina & Brasil & Chile & Colombia & México & Perú & América Latina \\
\hline IFI_2(t-4) & $\begin{array}{l}-0.0155 \\
(-0.83)\end{array}$ & $\begin{array}{l}-0.0029 \\
(-1.75)^{*}\end{array}$ & $\begin{array}{l}-0.0069 \\
(-2.60)^{* * *}\end{array}$ & $\begin{array}{l}-0.0354 \\
(-6.63)^{* * *}\end{array}$ & $\begin{array}{l}-0.0024 \\
(-1.90)^{* *}\end{array}$ & $\begin{array}{l}-0.0103 \\
(-0.96)\end{array}$ & $\begin{array}{l}-0.0026 \\
(-1.38)\end{array}$ \\
\hline $\operatorname{LN}(\operatorname{ICRG}(t-1))$ & $\begin{array}{l}-1.3889 \\
(-18.57)^{* * *}\end{array}$ & $\begin{array}{l}-0.5097 \\
(-10.12)^{* * *}\end{array}$ & $\begin{array}{l}-0.0504 \\
(-2.41)^{* * *}\end{array}$ & $\begin{array}{l}-0.0289 \\
(-0.53)\end{array}$ & $\begin{array}{l}-0.0986 \\
(-3.49)^{* * * *}\end{array}$ & $\begin{array}{l}-0.3369 \\
(-4.52)^{* * * *}\end{array}$ & $\begin{array}{l}-0.9743 \\
(-17.89)^{* * *}\end{array}$ \\
\hline $\operatorname{LN}(B M(t-1))$ & $\begin{array}{l}0.0093 \\
(1.14)\end{array}$ & $\begin{array}{l}0.0036 \\
(1.72)^{* *}\end{array}$ & $\begin{array}{l}0.0016 \\
(5.17)^{* * *}\end{array}$ & $\begin{array}{l}0.0066 \\
(2.34)^{* *}\end{array}$ & $\begin{array}{l}0.0014 \\
(0.84)\end{array}$ & $\begin{array}{l}0.0071 \\
(3.60)^{* * *}\end{array}$ & $\begin{array}{l}0.0035 \\
(1.85)^{*}\end{array}$ \\
\hline $\operatorname{LN}\left(M C_{t}\right)$ & $\begin{array}{l}-0.0089 \\
(-1.71)^{*}\end{array}$ & $\begin{array}{l}-0.005 \\
(-4.14)^{* * *}\end{array}$ & $\begin{array}{l}0.0031 \\
(1.18)\end{array}$ & $\begin{array}{l}-0.0027 \\
(-2.44)^{* * *}\end{array}$ & $\begin{array}{l}-0.0046 \\
(-3.62)^{* * *}\end{array}$ & $\begin{array}{l}-0.0072 \\
(-4.48)^{* * *}\end{array}$ & $\begin{array}{l}-0.0136 \\
(-4.72)^{* * *}\end{array}$ \\
\hline $\mathrm{LIQ}_{\mathrm{t}}$ & $\begin{array}{l}0.0204 \\
(1.46)\end{array}$ & $\begin{array}{l}0.0269 \\
(2.03)^{* *}\end{array}$ & $\begin{array}{l}-0.0001 \\
(-0.57)\end{array}$ & $\begin{array}{l}0.0009 \\
(1.04)\end{array}$ & $\begin{array}{l}0.0048 \\
(3.48)^{* * *}\end{array}$ & $\begin{array}{l}-0.0002 \\
(-0.56)\end{array}$ & $\begin{array}{l}0.0001 \\
(0.74)\end{array}$ \\
\hline INFt & $\begin{array}{l}0.4885 \\
(3.38)^{* * *}\end{array}$ & $\begin{array}{l}0.1404 \\
(1.33)\end{array}$ & $\begin{array}{l}0.0387 \\
(1.05)\end{array}$ & $\begin{array}{l}0.0622 \\
(0.71)\end{array}$ & $\begin{array}{l}0.1587 \\
(2.44)^{* * *}\end{array}$ & $\begin{array}{l}0.6698 \\
(3.77)^{* * *}\end{array}$ & $\begin{array}{l}0.4208 \\
(4.15)^{* * *}\end{array}$ \\
\hline Constante & $\begin{array}{l}6.3004 \\
(19.91)^{* * *}\end{array}$ & $\begin{array}{l}2.3571 \\
(11.21)^{* * *}\end{array}$ & $\begin{array}{l}0.2918 \\
(3.22)^{* * *}\end{array}$ & $\begin{array}{l}0.2771 \\
(1.23)\end{array}$ & $\begin{array}{l}0.6111 \\
(5.06)^{* * *}\end{array}$ & $\begin{array}{l}1.5949 \\
(5.06)^{* * *}\end{array}$ & $\begin{array}{l}4.6104 \\
(18.69)^{* * *}\end{array}$ \\
\hline No Observaciones & 1639 & 1393 & 1134 & 479 & 1432 & 541 & 6693 \\
\hline No de empresas & 61 & 57 & 57 & 23 & 41 & 31 & 270 \\
\hline Prob $>$ Chi2 2 & 0.0000 & 0.0000 & 0.0000 & 0.0000 & 0.0000 & 0.0001 & 0.0000 \\
\hline R2 & 0.2868 & 0.4346 & 0.6946 & 0.3708 & 0.5334 & 0.4802 & 0.3176 \\
\hline
\end{tabular}

La variable dependiente es el costo de capital propio (CE) estimado con el modelo de Damodaran (ecuación 2). La tabla muestra los resultados de la regresión de datos de panel (ecuación 7) para cada país y para toda la región Latinoamericana con el indicador de integración financiera que es el stock de activos y pasivos extranjeros como porcentaje del PIB $\left(I F I_{1}\right)$. Todas las regresiones se realizaron con efectos fijos y se corrigieron por heterocedasticidad y autocorrelación con Prais - Winsten. Las variables de control de riesgo país (ICRG), expectativas de los inversionistas (BM) y tamaño de las empresas (MC) fueron incluidas en sus transformaciones logarítmicas para suavizar las series. Los paréntesis reportan los estadísticos $Z$.

Nótese que para Latinoamérica y para cada uno de los seis países considerados este indicador es estadísticamente significativo y con el signo apropiado, es decir, un incremento del mismo implica una reducción del costo de capital propio. Asimismo, es interesante notar como este indicador requiere de seis meses de rezago (dos trimestres) para ser estadísticamente significativo.

Los resultados obtenidos coinciden con los obtenidos por Harvey (2004) y Kim y Singal (2000) los cuales mencionan que la mayor integración reduce los costos de capital debido a que los inversionistas exigirán una menor compensación a países o mercados financieros más integrados por haber eliminado el riesgo no sistemático de su inversión y que la apertura de los mercados de capitales produce una mejora en la eficiencia de la distribución del capital en los mercados domésticos.

Por otro lado, el riesgo país - LN (ICRG) - tiene una influencia significativa sobre los costos de capital propio en Latinoamérica y para todos los países, con excepción de Colombia. Esto muestra que a medida que aumenta el riesgo país (compuesto por el económico, financiero y político), los inversionistas exigen un mayor costo de capital propio al momento de realizar sus inversiones con un rezago de tres meses. 
Se encontró, al igual que Pointon (2004), Hail y Leuz (2006) y Chen, Chen y Wei (2009), que una disminución de las expectativas de rendimiento de los inversionistas - LN (BM) - medidas por un aumento en el ratio BM, generan aumentos de los costos de capital propio pero solo en Brasil, Chile y Colombia y con un trimestre de rezago. Asimismo se encontró que el tamaño de las empresas - LN (MC) - tiene una relación estadísticamente significativa y negativa con los costos de capital propio en todos los países, con excepción de Chile. Esto confirma la visión de que las empresas más grandes enfrentan un menor costo de capital propio debido a economías de escala en la obtención de financiamiento.

Según los resultados, aparentemente la liquidez accionaria (LIQ) responde de manera distinta en los mercados latinoamericanos, lo cual va en contra de lo inicialmente planteado. Para países como Chile y Perú, aunque no estadísticamente significativo, una mayor liquidez conlleva a que los inversionistas exijan un menor retorno por la acción debido a los menores costos de transacción asociados con dicho título, coincidiendo con los resultados obtenidos por Omran y Pointon (2004) y Jain (2005). Por otro lado en países como Argentina, Brasil, Colombia y México los mayores niveles de liquidez podrían conllevar a una mayor volatilidad de la acción, produciendo en última instancia una relación positiva entre estas dos variables.

De forma consistente con Hail y Leuz (2006) y Chen, Chen y Wei (2009), la inflación (INF) es un factor que incrementa los costos de capital propio en América Latina. Esto se debe a que la mayor parte de las empresas latinoamericanas listan sus acciones en moneda local y una mayor inflación reduciría el valor real de la moneda - y con ello su rentabilidad - lo que llevaría a los inversionistas a exigir un mayor retorno por las inversiones realizadas, aumentando con ello los costos de capital.

En la Tabla 5 se muestran los resultados obtenidos con el segundo indicador de integración financiera que es el ratio de flujos de inversión extranjera sobre el PIB $\left(I F I_{2}\right)$. En términos generales, se obtienen los mismos resultados con un año de rezago (cuatro trimestres). A pesar de ello, en todos los casos el segundo indicador de integración mantiene el signo negativo indicando que un incremento medido en términos del flujo de inversión extranjera como porcentaje del PIB disminuye los costos de capital propio. 
Tabla 5. Integración financiera y costos de capital propio en América Latina por países (2000-2009): $I F I_{2}$

\begin{tabular}{|c|c|c|c|c|c|c|c|}
\hline \multirow[b]{2}{*}{ Indicador } & \multicolumn{7}{|c|}{ Modelo de Efectos Fijos con errores Estándares Corregidos (Prais - Winsten) } \\
\hline & Argentina & Brasil & Chile & Colombia & México & Perú & América Latina \\
\hline IFI_2(t-4) & $\begin{array}{l}-0.0155 \\
(-0.83)\end{array}$ & $\begin{array}{l}-0.0029 \\
(-1.75)^{*}\end{array}$ & $\begin{array}{l}-0.0069 \\
(-2.60)^{* * *}\end{array}$ & $\begin{array}{l}-0.0354 \\
(-6.63)^{* * *}\end{array}$ & $\begin{array}{l}-0.0024 \\
(-1.90)^{* *}\end{array}$ & $\begin{array}{l}-0.0103 \\
(-0.96)\end{array}$ & $\begin{array}{l}-0.0026 \\
(-1.38)\end{array}$ \\
\hline $\operatorname{LN}(\operatorname{ICRG}(t-1))$ & $\begin{array}{l}-1.3889 \\
(-18.57)^{* * *}\end{array}$ & $\begin{array}{l}-0.5097 \\
(-10.12)^{* * *}\end{array}$ & $\begin{array}{l}-0.0504 \\
(-2.41)^{* * *}\end{array}$ & $\begin{array}{l}-0.0289 \\
(-0.53)\end{array}$ & $\begin{array}{l}-0.0986 \\
(-3.49)^{* * *}\end{array}$ & $\begin{array}{l}-0.3369 \\
(-4.52)^{* * *}\end{array}$ & $\begin{array}{l}-0.9743 \\
(-17.89)^{* * *}\end{array}$ \\
\hline $\operatorname{LN}\left(B M_{(t-1)}\right)$ & $\begin{array}{l}0.0093 \\
(1.14)\end{array}$ & $\begin{array}{l}0.0036 \\
(1.72)^{* *}\end{array}$ & $\begin{array}{l}0.0016 \\
(5.17)^{* * *}\end{array}$ & $\begin{array}{l}0.0066 \\
(2.34)^{* *}\end{array}$ & $\begin{array}{l}0.0014 \\
(0.84)\end{array}$ & $\begin{array}{l}0.0071 \\
(3.60)^{* * *}\end{array}$ & $\begin{array}{l}0.0035 \\
(1.85)^{*}\end{array}$ \\
\hline $\operatorname{LN}\left(M C_{t}\right)$ & $\begin{array}{l}-0.0089 \\
(-1.71)^{*}\end{array}$ & $\begin{array}{l}-0.005 \\
(-4.14)^{* * *}\end{array}$ & $\begin{array}{l}0.0031 \\
(1.18)\end{array}$ & $\begin{array}{l}-0.0027 \\
(-2.44)^{* * *}\end{array}$ & $\begin{array}{l}-0.0046 \\
(-3.62)^{* * *}\end{array}$ & $\begin{array}{l}-0.0072 \\
(-4.48)^{* * *}\end{array}$ & $\begin{array}{l}-0.0136 \\
(-4.72)^{* * *}\end{array}$ \\
\hline LIQ $t_{t}$ & $\begin{array}{l}0.0204 \\
(1.46)\end{array}$ & $\begin{array}{l}0.0269 \\
(2.03)^{* *}\end{array}$ & $\begin{array}{l}-0.0001 \\
(-0.57)\end{array}$ & $\begin{array}{l}0.0009 \\
(1.04)\end{array}$ & $\begin{array}{l}0.0048 \\
(3.48)^{* * *}\end{array}$ & $\begin{array}{l}-0.0002 \\
(-0.56)\end{array}$ & $\begin{array}{l}0.0001 \\
(0.74)\end{array}$ \\
\hline INFt & $\begin{array}{l}0.4885 \\
(3.38)^{* * *}\end{array}$ & $\begin{array}{l}0.1404 \\
(1.33)\end{array}$ & $\begin{array}{l}0.0387 \\
(1.05)\end{array}$ & $\begin{array}{l}0.0622 \\
(0.71)\end{array}$ & $\begin{array}{l}0.1587 \\
(2.44)^{* * *}\end{array}$ & $\begin{array}{l}0.6698 \\
(3.77)^{* * *}\end{array}$ & $\begin{array}{l}0.4208 \\
(4.15)^{* * *}\end{array}$ \\
\hline Constante & $\begin{array}{l}6.3004 \\
(19.91)^{* * *}\end{array}$ & $\begin{array}{l}2.3571 \\
(11.21)^{* * *}\end{array}$ & $\begin{array}{l}0.2918 \\
(3.22)^{* * *}\end{array}$ & $\begin{array}{l}0.2771 \\
(1.23)\end{array}$ & $\begin{array}{l}0.6111 \\
(5.06)^{* * *}\end{array}$ & $\begin{array}{l}1.5949 \\
(5.06)^{* * *}\end{array}$ & $\begin{array}{l}4.6104 \\
(18.69)^{* * *}\end{array}$ \\
\hline No Observaciones & 1639 & 1393 & 1134 & 479 & 1432 & 541 & 6693 \\
\hline No de empresas & 61 & 57 & 57 & 23 & 41 & 31 & 270 \\
\hline Prob>Chi2 & 0.0000 & 0.0000 & 0.0000 & 0.0000 & 0.0000 & 0.0001 & 0.0000 \\
\hline R2 & 0.2868 & 0.4346 & 0.6946 & 0.3708 & 0.5334 & 0.4802 & 0.3176 \\
\hline
\end{tabular}

La variable dependiente es el costo de capital propio (CE) estimado con el modelo de Damodaran (ecuación 2). La tabla muestra los resultados de la regresión de datos de panel (ecuación 7 ) para cada país y para toda la región Latinoamericana con el indicador de integración financiera que es el flujo de capital extranjero como porcentaje del PIB $\left(I F I_{2}\right)$. Todas las regresiones se realizaron con efectos fijos y se corrigieron por heterocedasticidad y autocorrelación con Prais - Winsten. Las variables de control de riesgo país (ICRG), expectativas de los inversionistas (BM) y tamaño de las empresas (MC) fueron incluidas en sus transformaciones logarítmicas para suavizar las series. Los paréntesis reportan los estadísticos $Z$.

El que el indicador de integrador financiera sea significativo en el cuarto rezago implica que se deben mostrar una tendencia creciente de influjo de capital extranjero para que exista una disminución en la rentabilidad exigida en el corto plazo producto de una mayor demanda por los títulos. Esta interpretación se ve reforzada con el hecho de que este segundo indicador de integración financiera es mucho más volátil que el primero (compárense los gráficos 1 y 2), con lo cual se requieren más periodos para efectivamente mostrar una tendencia.

Con respecto a las demás variables de control, destaca el hecho de que el ratio BM resulta además significativo para el Perú mostrándose de esta forma la naturaleza de corto plazo de este indicador. Con respecto al indicador de liquidez aparece Brasil y con respecto a la inflación esperada aparece México como coeficientes estadísticamente significativos.

\section{Conclusión}

Los resultados muestran que efectivamente una mayor integración financiera, ya sea como una variable acumulada o como un flujo, reduce el costo de capital propio en los países Latinoamericanos. No obstante, es de notar que se requiere de un periodo de rezago que va entre medio año y un año para que el efecto de una mayor integración financiera reduzca el costo de capital propio de los países. 
Como es esperable, este rezago es mayor en el caso del indicador acumulado (stock) que en el caso del indicador flujo.

Las diferencias entre los países se producen en la significancia de las variables de control, especialmente en el caso de las expectativas de los inversionistas (BM) y liquidez (LIQ). Las variables más consistentes en todos los países son las referidas al riesgo país (ICRG), al efecto tamaño (MC) y a la inflación (INF). En los tres casos, los signos de los coeficientes son los correctos y estadísticamente significativos, con pocas excepciones.

En general los resultados muestran que es beneficioso promover la integración financiera debido a que esto conlleva a una reducción de los costos de capital propio, lo cual a su vez favorecería la inversión en la región y el crecimiento económico. No obstante, para que se diseñen políticas de estímulo sectoriales es muy importante conocer las diferencias del efecto de integración financiera sobre los costos de capital propio en los diferentes sectores económicos. Este tema, ciertamente, constituye un tema para la agenda futura de investigación.

Otro tema de interés es la comparación empírica de los modelos para estimar el costo de capital propio. Específicamente, es importante saber si los modelos ex-ante (basados en expectativas de los analistas financieros y que utilizan el modelo de dividendos descontados) poseen un desempeño empírico superior a los modelos basados en el variantes del CAPM en estudios de los factores que afectan el costo de capital propio.

Muchos otros temas quedan pendientes, tales como saber si un buen gobierno corporativo de las empresas Latinoamericanas reduce el costo de capital propio o si las empresas Latinoamericanas con una buena responsabilidad social están sujetas a un menor costo de capital, pero estos y muchos otros temas más forman parte de una rica agenda de investigación.

\section{Bibliografía}

Adam K., T. Jappelli, A. Menichini, M. Padula and M. Pagano (2002). Analyze, Compare, and Apply Alternative Indicators and Monitoring Methodologies to Measure the Evolution of Capital Market Integration in the European Union, Centre for Studies in Economics and Finance, University of Salerno, pp 66.

Ameer R. (2007). Time-varying Cost of Equity Capital in Southeast Asian Countries. Asian Economic Journal, 21(2), pp. 207-238

Agenor P.R. (2003). Benefits and Costs of International Financial Integration: Theory and Facts. World Economy, 26(8), pp. 1089-1118.

Banco Mundial (2009). World Development Indicators, Website

Bekaert G., and C. Harvey (1995). Time Varying World Market Integration. Journal of Finance, 50(2), pp. $403-444$.

Bekaert, G., and C. Harvey (2000). Foreign Speculators and Emerging Equity Markets. Journal of Finance,55(2), pp. $565-613$

Bekaert, G., C. Harvey and R. Lumsdaine (2001). Dating the Integration of World Equity Markets. Journal of Financial Economics, 65(2), pp. 203 - 247

Bekaert G., and C. Harvey (2002). Research in Emerging Markets Finance: Looking to the Future. Emerging Markets Review, 3(4), pp. 429 - 448 
Bekaert G., and C. Harvey (2003). Emerging Markets Finance. Journal of Empirical Finance, $10(1)$, pp. $3-55$

Chen K., Z. Chen and J. Wei (2009). Legal Protection of Investors, Corporate Governance, and the Cost of Equity Capital. Journal of Corporate Governance, 15(3), pp. 273-289

Collins, D., and M. Abrahamson (2006). Measuring the Cost of Equity in African Financial Markets. Emerging Markets Review, 7(1), pp. 67 - 81

Damodaran A. (2003). Country risk and Company Exposure: Theory and practice. Journal of Applied Finance, 13(2), pp. 64-78

Erb C., C. Harvey, and T. Viskanta (1996). Expected Returns and Volatility in 135 Countries. The Journal of Portfolio Management, 22(3), pp. 46-58.

Estrada J. (2002) Systematic Risk in Emerging Markets: The D-CAPM. Emerging Markets Review, 3(4), pp. 365-379

Errunza V., and D. Miller (2000). Market Segmentation and the Cost of Capital in International Equity Markets. Journal of Financial and Quantitative Analysis, 35(4), pp. $577-600$

Fama E., and K. French (1992). The Cross-Section of Expected Stock Returns. Journal of Finance, 47(2), 427-465

Fama E.F., and K.R. French (1998). Value versus Growth: the International Evidence. Journal of Finance, 53(6), pp. 1975-1999.

Fernandez P., J. Aguirreamalloa, and L. Corres (2011). Market Risk Premium Used in 56 Countries in 2011: A Survey with 6,014 Answers. Working Paper, IESE Business School, Barcelona

Ferson W., and D. Lock (1998). Estimating the Cost of Capital through Time: An Analysis of the Sources of Error. Management Science, 44(4), pp. 485-500.

Fondo Monetario Internacional (2009). International Financial Statistics, Website

Fuenzalida D., S. Mongrut y M. Nash (2005) Riesgo país y riesgo soberano: concepto y medición. Revista Mexicana de Economía y Finanzas, 4(4), pp. 347-367.

Fuenzalida D. and S. Mongrut (2010). Estimation of Discount Rates in Latin America: Empirical Evidence and Challenges. Journal of Economics, Finance and Administrative Sciences, 15(28), pp. 7-43

Goedhart M., T. Koller and D. Williams (2002). The Real Cost of Equity, McKinsey on Finance, Autumn.

Harvey C. (2004). Country Risk Components, the Cost of Capital, and Returns in Emerging Markets. En Sam Wilkin Ed., Country and Political Risk: Practical Insights for Global Finance, Risk Books, pp. $71-102$

Hail L. and C. Leuz (2006). International Differences in the Cost of Equity Capital: Do Legal Institutions and Securities Regulation Matter? Journal of Accounting Research, 44(3), pp. $485-531$

Hausman J. (1978) Specification Tests in Econometrics. Econometrica, 46(6), pp. 1251-1271

Henry P. (2000) Stock Market Liberalization, Economic Reform, and Emerging Market Equity Prices. Journal of Finance, 55(2), pp. 529 - 564

Jain P. (2005). Financial Market Design and the Equity Premium: Electronic versus Floor Trading. The Journal of Finance, 60(6), pp. 2955 - 2985

Kim H., and V. Singal (2000). Stock Market Openings: Experiences of Emerging Economies. The Journal of Business, 73(1), pp. 25 - 66

Lane P.R., and G.M. Milesi-Ferretti (2003). International Financial Integration, IMF Working Papers, 03/86, Fondo Monetario Internacional

Lewis J. (2000). Two-Stage Approaches to Regression Models in which the Dependent Variable is based on Estimates, Working Paper, Princeton University

Mariscal J. and R. Lee (1993). The Valuation of Mexican Stocks: An Extension of the Capital Asset Pricing Model, Goldman Sachs, NY

Mongrut S., A. Paskevicius, P. Dubinskas, R. Kovalevskaja and D. Fuenzalida (2010) Estimating the Cost of Equity in the Baltic Region, Ekonomika, 89(3), pp. 40-54.

Omran M. y J. Pointon (2004). The Determinants of the Cost of Capital by Industry within an Emerging Economy: Evidence from Egypt. International Journal of Business, 9(3), pp 22 
Pomerleano M and X. Zhang (1999). Corporate Finance Fundamentals and the Behavior of Capital Market in Asia, In Financial Markets and Development, Eds. A. Harwood, R. Lita y M. Pomerleano, 57-117, Brookings Institution, Washington D.C.

Prasad E., K Rogoff, S. Wie and M. Kose (2003). Effects of Financial Globalization on Developing Countries: Some Empirical Evidence. Occasional Paper, No. 220, Fondo Monetario Internacional

Pretorius E. (2002). Economic Determinants of Emerging Stock Market Interdependence, Emerging Markets Review, 3, pp. 84-105

Vo, X.V. (2005). A New Set of Measures on International Financial Integration, Working Paper, Third Financial Markets Asia - Pacific Conference, Sydney

Walker E. (2003). Costo de capital para empresas reguladas en Chile. Monografía, Pontificia Universidad Católica de Chile, pp 44.

Wooldridge, J. M. (2002). Econometric Analysis of Cross Section and Panel Data, MIT Press, pp 776 